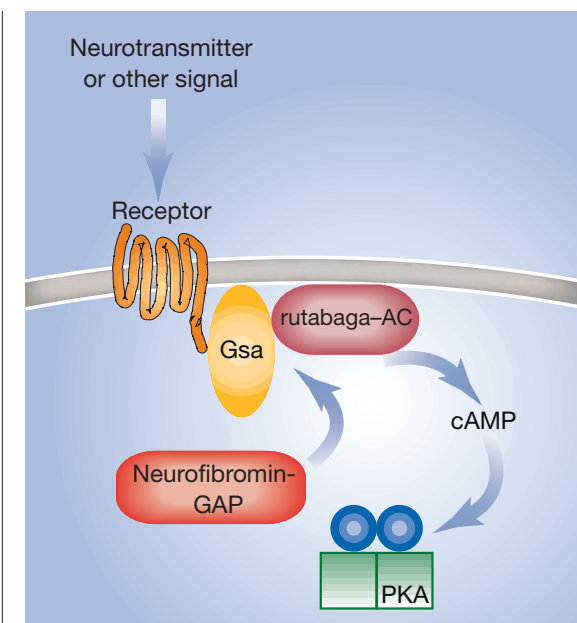

Figure 1 Neurofibromin signalling in learning. G-protein-coupled receptors are linked to adenylyl cyclase (AC) through the $\alpha$-subunit of the stimulatory G protein, Gs. Receptor activation stimulates adenylyl cyclase to synthesize cyclic AMP (cAMP) and activates the downstream effector, protein kinase $A$ (PKA). Guo et al. ${ }^{3}$ show that the neurofibromin protein is required in this pathway for normal odour learning in Drosophila, perhaps by direct interaction with the $G$ protein or with the adenylyl cyclase encoded by the rutabaga gene.

neurofibromin is needed in the mature brain for normal odour learning to occur.

But does neurofibromin involvement in learning depend on Ras? In Drosophila, the answer seems to be no. It was previously shown that a neuropeptide known as PACAP increases the activity of voltage-gated potassium channels ${ }^{8}$. This response involves the combined activation of the Ras pathway and the cyclic AMP pathway through adenylyl cyclase. Neurofibromin is required for this biological response to the neuropeptide but, surprisingly, the requirement lies not in the Ras pathway but in the cyclic AMP pathway, as application of cyclic AMP itself can restore the response in NF1 mutants9. So, in flies, neurofibromin is involved in the activation of adenylyl cyclase (Fig. 1).

Guo et al. ${ }^{3}$ have extended the experimental support for this role in three ways. First, they show that expression in transgenic flies of an active form of protein kinase A, a component of the cyclic AMP pathway, corrects the learning deficiency of NF1 mutants. Second, they find that neurofibromin is required for the normal activation of adenylyl cyclase activity by GTP. Finally, the magnitude of the deficiency in stimulation of adenylyl cyclase by GTP in NF1 mutants is similar to that in learning mutants of the rutabaga gene. As rutabaga is the structural gene for one type of adenylyl cyclase, it appears that Drosophila neurofibromin works specifically through this cyclase.

This adds up to suggest that neurofibromin in the fly has a biochemical function not yet discovered in mammals: positive regulation of the activation of adenylyl cyclase by GTP. Furthermore, this regulation takes place in mature neurons, and its failure in adults causes learning disabilities. But how might these findings apply to mammalian neurofibromin, NF1 patients and mammalian cognition?

It is now imperative to test whether Imammalian neurofibromin can interact with and activate adenylyl cyclase/G-protein complexes. There is plenty of room on the protein for direct interactions, as the GAP domain occupies only $10 \%$ of the sequence ${ }^{10}$. Furthermore, some alternatively spliced variants of mammalian neurofibromin are expressed only in mature neurons ${ }^{11}$, offering the potential for isoform-specific functions such as cyclase activation. Nevertheless, even though Drosophila neurofibromin is highly homologous to mammalian neurofibromin and has Ras-GAP activity in vitro ${ }^{12}$, it may be that the fly neurofibromin simply functions differently. Genetic attempts to show that Drosophila neurofibromin also negatively regulates Ras have failed ${ }^{12}$, perhaps owing to compensation by other GAPs, or perhaps because neurofibromin has nothing to do with Ras signalling in flies.

But can we really learn about human learning disabilities from the fly? After all, the fly has a small brain, limited intellectual capacity, and no higher cognitive traits such as executive function. It seems so, as the conservation between mammalian and Drosophila genes observed to date, even for those involved in behaviour, is remarkable. Several genes that are required for Drosophila learning, including dunce, rutabaga, DCO, CREB, Volado,13 and NF1 (ref. 14), are all involved in mammalian learning or other behaviours. Thus, if the parallels remain true and the learning disabilities of NF1 patients stem from signalling rather than developmental defects, then patient treatment with cognitive enhancers could be approached with optimism.

Ronald L. Davis is in the Department of Molecular and Cellular Biology and the Department of

Psychiatry and Behavioral Sciences, Baylor College of Medicine, Houston, Texas 77030, USA. e-mail: rdavis@bcm.tmc.edu

1. North, K. N. et al. Neurology 48, 1121-1127 (1997)

2. Ozonoff, S. Am. J. Med. Genet. 89, 45-52 (1999).

3. Guo, H.-F., Tong, J., Hannan, F., Luo, L. \& Zhong, Y. Nature 403, 895-898 (2000).

4. Gutmann, D. Arch. Neurol. 56, 1322-1323 (1999).

5. Moore, B. D., Slopis, J. M., Schomer, D., Jackson, E. F. \& Levy, B. M. Neurology 46, 1660-1668 (1996).

6. Dauwalder, B. \& Davis, R. L. J. Neurosci. 15, 3490-3499 (1995).

Grotewiel, M. S., Beck, C. D., Wu, K. H., Zhu, X. R. \& Davis, R. L. Nature 391, 455-460 (1998).

8. Zhong, Y. Nature 375, 558-592 (1995).

9. Guo, H.-F., Inge, T., Hannan, F., Bernards, A. \& Zhong, Y. Science 276, 795-798 (1997).

10. McCormick, F. Curr. Opin. Genet. Dev. 5, 51-55 (1995).

11. Gutmann, D. H., Zhang, Y. \& Hirbe, A. Ann. Neurol. 46, 777-782 (1999)

12. The, I. et al. Science 276, 791-794 (1997).

13. Davis, R. L. Physiol. Rev. 76, 299-317 (1996).

14. Silva, A. J. et al. Nature Genet. 15, 281-284 (1997).
Daedalus

\section{Grinding waste away}

How to get rid of domestic and urban rubbish? Recycling is troublesome, incineration unpopular, and composting impeded by the presence of plastic and metal. Landfill merely entombs the trash archivally for future archaeologists. One answer might be to feed the stuff to pet goats; they eat anything, and would generate useful metabolic heat from its digestion. Unlike a passive compost heap or landfill, an animal gut is ceaselessly churning. Some animals swallow stones or grit to aid their digestion; these must help the process mechanically or chemically. In this connection, Daedalus recalls that halocarbons can be decomposed by ballmilling them with lime. Mechanical impact and abrasion actually break up the molecules and encourage their reaction.

So Daedalus is designing a mechanically agitated composter for all our rubbish. Its steady churning and pounding, shearing the molecules at the point of impact whenever two chunks of rubbish come together, will degrade its contents at an unprecedented rate. Even plastics will be rapidly oxidized to products edible by the resident bacteria. Chemical, mechanical, thermal and biological decomposition will all proceed in parallel. Too violent an agitation might squash the bacteria faster than they could multiply, but slower rates should be entirely effective. Adding domestic sewage to the mix would solve another disposal problem at the same time.

A large-scale rubbish composter would be a long inclined cylinder rotating on its axis. Rubbish added continuously at the elevated end would work its way slowly down; the cans and bottles in it would act as grinders for the organics. Its inlet zone would be kept anaerobic, to generate useful methane. Downstream, injected air would switch it to aerobic decomposition. Toxins and pollutants would be totally mineralized by this steady mechanochemical battering. Only rust, glass and ceramic grit would emerge at the far end.

But Daedalus wants to abolish rubbish collection as well. So he plans a simpler domestic composting dustbin, to degrade the rubbish and sewage of one household. It will grind away outside the house, but will be plumbed into the heating system to contribute useful warmth to the domestic interior.

David Jones

The Further Inventions of Daedalus (Oxford University Press), 148 past Daedalus columns expanded and illustrated, is now on sale. Special Nature offer: m.curtis@nature.com 